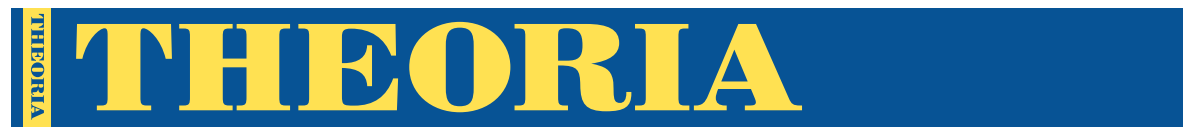

THEORIA, 2009, 75, 344-357

doi:10.1111/j.1755-2567.2009.01048.x

\title{
On the Modal Content of $A$ Posteriori Necessities
}

by

\author{
TUOMAS E. TAHKO \\ Durham University
}

Abstract: This article challenges the Kripkean interpretation of a posteriori necessities. It will be demonstrated, by an analysis of classic examples, that the modal content of supposed a posteriori necessities is more complicated than the Kripkean line suggests. We will see that further research is needed concerning the a priori principles underlying all a posteriori necessities. In the course of this analysis it will emerge that the modal content of a posteriori necessities can best be described in terms of a Finean conception of modality - by giving essences priority over modality. The upshot of this is that we might be able to establish the necessity of certain supposed a posteriori necessities by a priori means.

Keywords: a posteriori, a priori, necessity, possibility, Kripke, modality, essences.

\section{The Traditional Account}

The traditional, widely accepted story about $a$ posteriori necessities goes like this: an empirical discovery associated with a supposed a posteriori necessary statement together with a certain general a priori principle imply that an identity statement is an a posteriori necessity. This story comes of course from Saul Kripke (1980, p. 109), although presently we are not concerned about what Kripke himself might think about these matters. In any case it is clear that there is an interpretation of Kripke which goes as suggested above and seems to be fairly widely accepted amongst Kripkeans. ${ }^{1}$

What we want to ask now is: what is the modal content of a posteriori necessities based on; in virtue of what are they necessary? At least in most cases, the modal content seems to come from the a priori part. To take one of the usual examples, consider the identity statement "Hesperus is Phosphorus". We can analyse it as follows. We know a posteriori that Hesperus is in fact Phosphorus, that is, we know that the identity statement "Hesperus is Phosphorus" is true. Further, we know $a$ priori that if Hesperus is Phosphorus, then it is necessary that Hesperus is Phosphorus. Clearly, Hesperus is necessarily Phosphorus in virtue of the a priori part.

Even if this story were correct, more needs to be said in its support. Kripke does not seem to be particularly concerned about this, but his commentators have attempted to fill the gaps. One issue that is especially pressing concerns the

1 See, for instance, Hughes (2004) or Soames (2005). 
empirical discoveries associated with a posteriori necessities, i.e., how do we know that an identity statement is in fact true? Christopher Hughes suggests the following concerning "Hesperus is Phosphorus":

We know that Hesperus is a celestial body and that Phosphorus is a celestial body. Also, given what we know about where Hesperus and Phosphorus are at certain times, and about how celestial bodies move, we can infer that Hesperus and Phosphorus are in some of - indeed, in all of - the same places at all the same times. And we know that different celestial bodies don't occupy all the same places at all the same times. So we know that Hesperus and Phosphorus are the same celestial body, and thus that Hesperus $=$ Phosphorus is true (Hughes, 2004, p. 213; footnote omitted).

This analysis, it seems, is very much on the right lines. However, it complicates matters somewhat, for now it seems that further modal content is introduced to the story before the Kripkean proof of the necessity of identity enters the picture. Namely, we know that different celestial bodies do not occupy all the same places at all the same times. This information concerns the essential features of celestial bodies, and it is crucial for the empirical discovery that Hesperus is Phosphorus. Furthermore, it seems that the modality involved here is of a different kind than the modality involved in the a priori truth that if an identity statement is true it is necessarily true. This is because the latter is based on the logical proof familiar from Kripke (1971) and, if true, expresses something general about identity statements. This logical proof, however, amounts to little unless further assumptions are introduced. Specifically, its applicability may be restricted to identity statements concerning singular terms (cf. Soames, 2005, pp. 23-24), yet examples concerning theoretical identity statements such as "Water $=\mathrm{H}_{2} \mathrm{O}$ " are generally considered to be the most important ones. Furthermore, the modality appears to be attached to all identity statements simply because they are identity statements, even though there are important discrepancies between the identity conditions of different kinds of entities. Indeed, it would appear that the modality in question is logical in nature, whereas the modality in the empirical finding is metaphysical: it concerns the essences of the entities under scrutiny, it expresses something metaphysically substantial. What is the difference between these types of modality? Well, logical necessity, which I take to be a subspecies of metaphysical modality, does not entail metaphysical necessity. ${ }^{2}$ On reflection, I do not think that this is a very controversial point: we have a number of alternative logics with different inference rules and hence we can have different results concerning what is necessary. Unless we have some reason to think that the logic we use corresponds with reality, then there is no

2 At least insofar as we are dealing with so-called strict or narrow logical necessity, i.e., necessity in virtue of the laws of logic and necessity in virtue of the laws of logic plus the definitions of concepts, respectively. So-called broad logical necessity, which takes into account the natures of entities, is effectively metaphysical necessity. 
direct entailment from logical modality to metaphysical modality. Indeed, if "Water $=\mathrm{H}_{2} \mathrm{O}$ " is a necessary truth, then this necessity is grounded in a metaphysical truth, not a logical one, as we will see in the course of this paper. In any case, even if there is a way for the Kripkean to overcome these problems, the question of the additional modal content within the empirical discovery remains.

\section{The $A$ Priori Content that Precedes Empirical Discoveries}

What is the source of the additional modal content in the empirical discovery? If we look at the passage quoted from Hughes above, it appears that he is listing some essential features of celestial bodies, planets. Moreover, the modal content of the empirical discovery seems to be grounded in these essential features, as it is exactly in virtue of the impossibility of celestial bodies occupying all the same places at all the same times that we know Hesperus and Phosphorus to be identical. In other words, we need a priori knowledge about the general essence of the kind "planet" to be able to derive that Hesperus is necessarily Phosphorus. ${ }^{3}$

Perhaps we ought to look deeper into this a priori part, as the apriority of the fact that two material bodies cannot occupy the same space-time point could be contested. It could be argued that this is an empirical discovery, perhaps based on Coulomb's law ${ }^{4}$ and the fact that material particles are subject to this law. But a closer examination reveals once again that there is a priori content sneaked in with the empirical discovery. Coulomb's law is a generalisation and it is indeed based on empirical observations, but if it is to carry any modal content, namely to imply that material bodies of the same kind are necessarily identical if they occupy the same space-time point, something more must be said. Although Coulomb's law seems to have an extremely wide applicability, empirical experiments can never verify its universal applicability. Accordingly, we need to combine Coulomb's law with a theory of electrostatic interaction. Furthermore, there are even more fundamental laws in effect here, specifically the Pauli Exclusion Principle, ${ }^{5}$ which is ultimately responsible for the space-occupying effect of all material particles. There are, however, particles that are not subject to the Pauli Exclusion Principle, namely bosons.

3 Furthermore, it is of course assumed here that Venus is a planet and that "Hesperus" and "Phosphorus" are directly referential. Additionally, it may be that the essential features are knowable (only) a priori, as Lowe (2007) argues. We will consider this in due course. This has also been discussed in Tahko (2008).

4 Coulomb's law concerns the relations between electric charges, namely, like chargers repel and unlike chargers attract.

5 The Pauli Exclusion Principle states that no two identical fermions can have the same quantum number at the same time. 
All that has been said above is interesting, but does it have a genuine bearing on the identity conditions of massive material bodies, such as planets? I think not: naturally we are interested in the microphysical story in the background because the macrophysical story that we are primarily interested in presumably supervenes on the microphysical, but we do not need to tell the microphysical story in every instance. Regardless of whether there are particles that violate Coulomb's law or the Pauli Exclusion Principle, we know that material bodies of the kind "planet" are most certainly subject to these laws and it is no doubt essential to material bodies of this kind that they occupy space. We do not have a priori access to the laws of physics, so we can only know a posteriori that planets are subject to certain laws. However, I think that we do know a priori that entities of the kind "planet" occupy space, indeed, that this is essential to them. The purpose of this discussion is simply to demonstrate that the empirical issues concerning these matters are quite complicated, but perhaps we should be more interested in what is essential to the kind "planet". Accordingly, the a priori content that precedes the empirical finding that Hesperus is Phosphorus does not reduce all the way to quantum mechanics, although there is further a priori content also in the microphysical story.

\section{Further Complications: the Status of Essences and $\boldsymbol{A}$ Priori Principles}

Our analysis of a posteriori necessity hints towards an interpretation of metaphysical modality in terms of essences. As Kit Fine puts it, "we should view metaphysical necessity as a special case of essence" (1994, p. 8), rather than the other way around. Grounding metaphysical modality in essences does not only enable us to explain the modal content in empirical discoveries associated with a posteriori necessities, but also highlights the complexity of identity statements that are usually considered to be a posteriori and necessary. However, it appears that establishing the necessity of a given identity statement might not be quite as easy as the Kripkean line suggests. Theoretical identity statements, such as "Water = $\mathrm{H}_{2} \mathrm{O}$ ”, are especially interesting in this regard. Consider how Scott Soames explains their necessity: ${ }^{6}$

The [Soames-type Kripkean] account holds that "water" is a non-descriptive, directly-referential term designating a substance - where substances are taken to be physically constitutive kinds (instances of which share the same basic physical constitution). It is further assumed that a kind of this sort may have different instances in different world-states, and that if $a$ and $b$ are kinds with the same instances in all possible world-states, then $a$ is $b$. These are clearly metaphysical assumptions, to which we add the natural corollary that for any substance, $s$, if, in some possible world-state,

6 There is an ongoing debate between Soames and E. J. Lowe about the necessary a posteriori; the quoted passage is from Soames' reply to Lowe - we will attempt to get into the bottom of this debate. 
instances of $s$ have a certain molecular structure, then instances of $s$ have that structure in every world-state. In other words, we assume that it is an essential property of a substance that instances of it have the molecular structure they do. From this it follows that ["Water $=\mathrm{H}_{2} \mathrm{O}$ "] is necessary if true, and that " $\mathrm{H}_{2} \mathrm{O}$ " - which I take to be equivalent to "the substance instances of which have a molecular structure with two hydrogen atoms and one oxygen atom" - is a rigid designator. Being true, ["Water $=\mathrm{H}_{2} \mathrm{O}$ "] is, therefore, necessary. Since knowing the proposition it expresses requires knowing of a certain substance that its instances have a particular chemical structure, ["Water = $\mathrm{H}_{2} \mathrm{O}$ "] is knowable only a posteriori (Soames, 2007, p. 36; footnotes omitted).

Apparently, the modal content of the supposed $a$ posteriori necessity "Water = $\mathrm{H}_{2} \mathrm{O}$ " is based on the assumption that substances, such as water, essentially have the molecular structure that they do and therefore water has the molecular structure $\mathrm{H}_{2} \mathrm{O}$ in all possible worlds. What has been said in support of this assumption? ${ }^{7}$ Nothing, as far as I can tell. To say generally of substances that they essentially have the molecular structure that they do is a very strong claim indeed. Yet Soames gives no explicit support for the assumption. Take the case of water and $\mathrm{H}_{2} \mathrm{O}$. What can be said in support of the assumption that water essentially has the molecular structure it in fact does? Clearly, no amount of research into the chemistry of water will settle the matter, because we can only study the actual molecular structure of water. The claim is, however, that the organisation of hydrogen and oxygen atoms in water is necessary. It could be suggested that the general principle, that substances have their molecular structures essentially, is only a derivative of actual cases, but in this case the principle would hardly be a priori. Alternatively, perhaps the principle is not true of all substances, but rather confined to certain sorts of natural kinds. Be that as it may, something needs to be said in support of this.

We may attempt to trace the modal content here more rigorously. Let us consider the general case, which is what Soames seems to support. What would the empirical implications be like? Well, we know that the manner in which chemical compounds are formed is directly dependent on the atomic structure of the atoms involved, which naturally involves their electron configuration. Further, we know that the electron configuration depends on the energy levels of specific electrons and is moderated by the Pauli Exclusion Principle. Very quickly, this regress goes all the way down to fundamental forces, which are ultimately responsible for the binding of atoms. Accordingly, the claim that substances have their molecular structure essentially implies that the laws of physics governing the binding of atoms are metaphysically necessary. It is unsettling that something as widely acknowledged as the necessity of "Water $=\mathrm{H}_{2} \mathrm{O}$ " commits us to such strong claims.

Perhaps it is a physical necessity that substances have the particular molecular structure that they actually have, but it would be precarious to claim, without argument, that the fundamental forces could not have been arranged otherwise to

7 Earlier concerns about these issues have been raised by Salmon (2005) and Bealer (1987). 
produce a substance exactly like water in every detail, apart from the molecular structure. It is not clear how we are supposed to decide whether this substance would be water or not, and the claim at hand does not concern only water, but all substances. Certainly, something must be said about the general essence of substances to make sense of this claim, namely whether or not it is an essential feature of substances that they have the particular molecular structure that they actually have. This is a subject that is of course beyond empirical research, but more importantly: empirical research concerning substances would not be possible in the first place if we did not have at least some a priori knowledge about the general essence of substances. ${ }^{8}$

We may have a strong intuition about the essentiality of composition for substances, but the typical Kripkean story about these matters takes this as given, when clearly the source of this intuition should be our primary interest. In what follows I will suggest that this intuition, if it is valid, must be based on a priori reasoning. There have been some attempts (e.g., Salmon, 2005) to examine the source of this intuition before, but it seems to me that they have been half-hearted at best. Some guidelines for how we should proceed in this regard will be suggested in the last section of this article.

It may seem that what has been said does not pose a challenge to the Kripkean (or Putnamian), as the Twin-Earth story presumably allows for substances, such as "XYZ", that are superficially similar to water, but differ in molecular structure. It is just that a substance like this is not water, or so the story goes. But what is the argument for this assumption? As we saw, Soames certainly gives us no argument for it. It seems to be simply built in the account that we have the intuition that XYZ would not be water. A potential line of thought that might support this assumption is the one that was sketched above, but that line of thought seems to be unable to account for the necessity of "Water $=\mathrm{H}_{2} \mathrm{O}$ ", because the assumption that substances essentially have the molecular structure that they actually have is not supported. But even if there were an appropriate a priori argument available we would have reasons to doubt the route by which the necessity of "Water $=\mathrm{H}_{2} \mathrm{O}$ " was established, as Lowe (2007) has convincingly argued. ${ }^{9}$

Similar problems emerge with other classic examples, such as "Gold is the element with the atomic number 79". Do elements essentially have the atomic number that they actually have? Perhaps alternative physics which produces the periodic table with atomic numbers different from the actual ones, yet elements indistinguishable from the actual ones is metaphysically possible - perhaps not. In

8 The relationship between a priori inquiry and empirical research has been discussed extensively in Tahko (2008).

9 These reasons are derived from the Finean understanding of essence, namely that it should not be treated as a special case of metaphysical necessity. I take this point and develop it in what follows. 
any case, this is not something that can be settled by considering Twin-Earth scenarios. Rather, what we need is research concerning the essences of substances and elements.

\section{A Sideline: How the Account at Hand Differs from the Deflationary Line}

The upshot of the previous section is that we should take the essences of whichever entities we are concerned with more seriously than the classic Kripkean line does, but the shortcomings of the Kripkean line have also inspired completely opposite reactions. It should be made clear that the account being proposed here is not at all similar to the deflationary line suggested by Alan Sidelle and others. Sidelle analyses identity statements such as "Water $=\mathrm{H}_{2} \mathrm{O}$ " as follows: ${ }^{10}$

[E]ach necessary a posteriori truth should be seen as derived from a combination of an analytic principle of individuation that has empty spaces to be filled in by empirical findings and a particular empirical finding that of itself carries no modal weight. For example, in the case of water's being necessarily $\mathrm{H}_{2} \mathrm{O}$, the analytic principle might be "Nothing counts as water in any situation unless it has the same deep explanatory features (if any) as the stuff we call 'water' ", and the empirical fact, which makes the result a posteriori, is that the deep explanatory feature of the stuff we call "water" is being composed of $\mathrm{H}_{2} \mathrm{O}$ (Sidelle, 2002, p. 319).

Sidelle goes on to suggest that there is nothing metaphysical in the modal content of a posteriori necessities; instead we are dealing with analytic, linguistic principles. Given what has been said above, it is not hard to contest Sidelle's line. The supposed a priori content in a posteriori necessities can indeed be interpreted roughly like Sidelle, as well as Jackson (1998) and Chalmers (1996), suggest. However, we have already seen that the empirical part is not empty of modal content, i.e., that empirical inquiry does carry modal weight, as it involves a priori knowledge concerning at least the general essences of whatever kinds of entities we happen to be dealing with. ${ }^{11}$ For Sidelle and others, the focus seems to be on the analytic a priori principle which is supposedly responsible for the modal content of a posteriori necessities. Consequently, when Sidelle (as well as Jackson and Chalmers) talk about the a priori part in a posteriori necessities, they are talking about something that is analytic or linguistic in nature. The a priori part that we have been referring to is within the empirical, a posteriori part of the story, it

10 Sidelle refers, quite correctly, to Frank Jackson (1998) and David Chalmers (1996) in this connection - they take a somewhat similar line regarding a posteriori necessities.

11 Hence, I think that scientists (and everyone else) have a priori knowledge of general essences. Our epistemic access to essences is, I believe, based on our knowledge of metaphysical possibilities, which we can analyse with the help of a priori reasoning. However, it is not possible to provide a sufficient account of the epistemology of essences here; I merely wish to point out that we must have some kind of a priori access to essences to be able to explain metaphysical necessities. Accordingly, we are certainly not dealing with linguistic or conceptual knowledge here, but rather the most fundamental kind of metaphysical knowledge. 
precedes the empirical discovery. ${ }^{12}$ However, it is not my purpose here to refute or examine the deflationary views; it will be sufficient to note that they are in the opposite end of the scale.

\section{A Priori Instead of $\boldsymbol{A}$ Posteriori Metaphysically Necessary Truths?}

The quarrel that I have with the Kripkean line is thus altogether different from the Sidelle-Chalmers-Jackson line; it concerns the nature of essences and their role in the supposed a posteriori necessities. Lowe sums this up as follows:

If Soames is right, the key contribution of Kripke to the metaphysics of essence was to show how, by combining a priori knowledge of general essential truths with a posteriori knowledge of particular actual facts, we could generate instances of particular essential truths that are knowable only a posteriori (Lowe, 2007, p. 291).

Lowe goes on to suggest that it is more likely that essential truths are knowable only a priori if they are knowable at all. In fact, it appears that the cart has been put before the horse here: it looks as if particular facts, e.g., that water is in fact $\mathrm{H}_{2} \mathrm{O}$, are the reason for the intuitions concerning general essential truths, e.g., that substances have their actual molecular structures essentially - or, at any rate, the $a$ priori inquiry that led to this general essential truth has not been made explicit. Accordingly, the move to particular essential truths, e.g., that water has its molecular structure essentially, seems unwarranted even within the Kripkean scheme. However, our main concern is that the Kripkean scheme is misleading to start with. An alternative account, on the lines of Fine and Lowe (although Fine seems to be neutral regarding our epistemic access to essences), would be to give the priority to essences, in which case a priori inquiry into the essences of the entities under investigation is enough - a posteriori knowledge of particular actual facts need not enter the picture. It would indeed appear that, at least in some cases, supposed $a$ posteriori necessities are not a posteriori at all, but rather a priori. Lowe (2007) also questions whether particular substances, such as water, even have individual essences. It is thus safer to illustrate the scheme with the help of another example, preferably one that does not concern individual essences. I will now proceed to analyse the details of the a priori work that we need to engage in to establish the necessity of some common examples of supposed a posteriori necessities.

Consider the classic example "Cats are Animals". Nathan Salmon has provided a Kripkean analysis of this and related examples:

We know a priori that if a biological kind (e.g., a species) $k$ is subsumed under a higher-level biological kind (e.g., a genus, class, kingdom, etc.) $k^{\prime}$, then it is necessary that $k$ is subsumed under

12 It should be mentioned in this connection that the decoupling of a priori and a posteriori content here is somewhat misleading, as has been demonstrated in Tahko (2008). 
$k^{\prime}$. We also know by the direct reference theory of the designation of natural kind terms that such terms as "cat", "tiger", "mammal", and "animal" are rigid designators of natural kinds. Putting these two together, we know a priori, by "philosophical analysis," that if all cats are animals, then it is necessary that all cats are animals, and if all tigers are mammals, then it is necessary that all tigers are mammals, etc. Science discovers empirically that cats are in fact animals, and that tigers are in fact mammals. Combining these scientific discoveries with what we know a priori by philosophical analysis, we infer that it is necessary, even though a posteriori, that cats are animals and that tigers are mammals. Given what we know by philosophical analysis - the theory of direct reference plus the a priori essentialist fact that every biological kind $k$ is such that it could not fail to be subsumed under any of the higher level biological kinds $k^{\prime}$ that in fact subsume it - any empirical discovery that cats are in fact animals, or that tigers are in fact mammals, is indirectly but automatically an empirical discovery that it is necessary that cats are animals, or that tigers are mammals (Salmon, 2005, p. 195).

Leaving aside for the moment the problems involved with natural kind terms and whether "animal" even constitutes a natural kind - it seems clear that the $a$ priori content concerning the supposed a posteriori necessity "Cats are Animals" includes knowledge about the necessary connection between a higher-level category and a lower-level category, i.e., a category and its subcategory. However, it is less clear what the status of the empirical discovery that cats are in fact animals is. An alternative analysis of the example reveals that we might be able to establish the necessity of "Cats are Animals" entirely by a priori means. If we have a priori access to categorial information, which information concerning the category of "Animals" presumably is, then we can grasp the general essence of the category of "Animals". Similarly, we can grasp the general essence of the kind "Cat", and it is surely part of the general essence of the kind "Cat" that it is a subcategory of "Animals". ${ }^{13}$ In other words, simply by the a priori information concerning the necessary connection between a category and its subcategory, and by the a priori information concerning the general essences of "Cats" and "Animals", namely that the first is a subcategory of the latter, we can derive the necessity of "Cats are Animals". The empirical discovery that cats are in fact animals does not enter the picture, contrary to how the Kripkeans would have it. This seems quite plausible, because even describing what the empirical discovery that cats are in fact animals is appears to be problematic. To establish that cats are animals, we must already know what animals are, that is, we must have grasped the general essence of "Animals". Furthermore, before we can engage in any empirical research concerning cats, we must also have grasped the general essence of "Cats". Thus, we could discover, by empirical means, that the creatures we thought were cats are in fact

13 Why think that we would have a priori access to categorial information? Well, for one thing, it seems that we can have information about merely possible kinds of entities, and this information could not be $a$ posteriori (because there are no entities of that kind in the actual world). Much more would need to be said about this of course, but I only hope to hint towards an alternative analysis of what is going on in supposed cases of a posteriori necessity. This type of approach has also been hinted towards by Lowe (e.g., 2007). 
demons, but this only means that in the actual world there are no cats, but only "fool's cats". ${ }^{14}$ It would still be the case that "Cats are Animals" is a necessary a priori truth - in all possible worlds where cats exist, they are animals.

One concern that might emerge at this point is that "Animal" is vague: are for instance viruses animals? ${ }^{15}$ Perhaps it is even a part of the essence of "Animal" that it is vague. As I noted above, I do not wish to engage in a thorough analysis of "animal" here; perhaps it does not even constitute a natural kind. However, although there is clearly vagueness over natural kind terms, I think that we are here dealing with linguistic rather than metaphysical vagueness. In general, we might be wrong about what the identity and existence conditions of certain natural kinds are, or mistaken about a natural kind altogether, but this does not imply that there has to be metaphysical vagueness over the matter. Accordingly, we can and often do make mistakes; even if we have a priori access to the essences of natural kinds, it does not mean that we always get the story right. The point of this article is to underline this.

A further analysis of the epistemic side of the story might be helpful, although I have discussed it in more detail elsewhere (cf. Tahko, 2008). Firstly, the notion of "a priori" is not being used in its traditional sense here, although for current purposes it might be enough to note that we are dealing with synthetic rather than analytic a priori knowledge. It is also crucial for this account that information about essences precedes empirical information. The link between our a priori capabilities and knowledge about essences is metaphysical modality: a priori reasoning concerns metaphysical modality and metaphysical modality is grounded in essences, as the Fine-Lowe line suggests. So, "Cats are Animals" could be considered an a priori metaphysical necessity in virtue of:

1. A priori knowledge concerning the necessary connection between a category and its subcategory, namely that a category has its subcategories by necessity.

2. A priori knowledge concerning the general essence of "Animals", namely that "Animals" is a categorial term capable of having certain types of subcategories, i.e., instances of "Animals".

3. A priori knowledge concerning the general essence of "Cats", namely that it is metaphysically possible that there are creatures such as cats which satisfy the criteria for a subcategory of "Animals", i.e., they are instances of animals.

Perhaps the most interesting part is the third one. We only need a priori knowledge about the metaphysical possibility of a kind such as "Cats" to be able to grasp the general essence of "Cats". Once we have the general essences of "Cats" and

14 Kripke was right about this.

15 Thanks to an anonymous reviewer for this point. 
"Animals", we only need to add the further piece of a priori knowledge concerning categories and subcategories to be able to determine that "Cats are Animals" is an a priori metaphysical necessity. We do not need to refer to actual cats or animals at all; the metaphysical necessity holds regardless of the status of cats and animals in the actual world, even if there are no such entities.

So, it appears that we can provide an analysis of the necessity of "Cats are Animals" which does not rely on empirical information, but how about "Tigers are Mammals"? It might seem that it is less plausible that the empirical discovery that tigers are in fact mammals does not enter the picture, although the case appears to be analogous to "Cats are Animals" - it certainly is for Salmon. However, it is questionable whether "Mammal" behaves in the same manner as "Animal". My Oxford English Dictionary defines "Mammal" as a warm-blooded vertebrate animal, which has hair or fur, produces milk and typically gives birth to live young. These features, it seems, are contingent: could tigers not evolve in such a manner that they cease to fulfil the criteria of "Mammal"? Accordingly, perhaps we are not dealing with a natural kind here at all, and perhaps "Tigers are Mammals" is indeed a posteriori, but also contingent. This highlights the fact that we must be particularly sensitive to the subject-matter of the identity-statements that we are dealing with, they must receive individual treatment. As I noted above, we could easily be altogether mistaken about some natural kinds; this may even be the case with "Animals", which makes the study of the essences of whichever entities we are dealing with all the more important.

We can now also reconsider the case of "Water $=\mathrm{H}_{2} \mathrm{O}$ ". We would need at least the a priori information that substances have their molecular structure by necessity, i.e., information concerning the general essence of chemical substances, to be able to determine that water molecules are necessarily composed of two hydrogen atoms and one oxygen atom - this much is uncontroversial. However, as we saw, it is far from obvious what the general essence of chemical substances includes. It may be that there is nothing more to the story in addition to the a priori principles concerning chemical substances: unless water has a further, individual essence, we can establish the necessity of water being $\mathrm{H}_{2} \mathrm{O}$ simply with the help of the general essence of chemical substances. Of course, it is also an open question whether water does have an individual essence. In any case, if the appropriate a priori arguments can be established, then similar analysis would be available in the case of "Water $=\mathrm{H}_{2} \mathrm{O}$ " as we saw above in the case of "Cats are Animals". It appears though that such arguments are extremely difficult to establish. However, I admit that I share the intuition that chemical substances have their molecular structure essentially, and I would like to examine the origins of this intuition briefly.

In section 3 we observed that if this intuition is valid, then it must be a physical necessity that chemical substances have the very molecular structure that they in fact have. How could we support this claim? Well, the forming of molecules is 
dependent on the bonding ability of atoms, a crucial feature of which is the electron configuration of atoms. As we have already noted, this is moderated by the Pauli Exclusion Principle. Now, the question is whether we could have a different set of atoms, call it XYZ, which could produce the chemical substance water. Note again that the Twin-Earth scenario does not have a bearing on this: we are looking for a justification for the intuition on which the whole scenario is based (at least when it is interpreted as expressing something metaphysically substantial, as serious essentialists would have it). It seems thus that our intuitions underlying the Twin-Earth scenario and related examples have not been sufficiently analysed in this regard. As has already been argued, the intuition that chemical substances have their molecular structures essentially implies that certain laws of physics are necessary. But this is a very controversial assumption - it appears that some worlds with different laws of physics are ruled out as impossible automatically. Now, if they are indeed impossible, surely this requires a more detailed discussion.

Perhaps it should also be noted that even though water appears, on the face of it, to be a non-functional, compositional stuff, ${ }^{16}$ what we are really interested in are its chemical properties rather than its composition. That is, if we had a substance that has exactly the same chemical properties as water but a different molecular composition, would we really be entitled to say that it is not water? As I have stressed above, I do not think that this has been established. However, if it is indeed a physical necessity that chemical substances have their molecular structures essentially, then we simply could not have a substance that has the same chemical properties as water, but a different molecular composition. Accordingly, the intuition that XYZ would not be water, if it is a valid intuition, is based on the fact that there could not be XYZ! It is an interesting question whether the intuition really is valid. It certainly seems that in the actual world there could not be a combination of atoms XYZ such that it would replicate the chemical properties of water, although we would have to turn to our colleagues in physics and chemistry for the details. But it is up to philosophers to determine whether the situation could be different in other possible worlds - our colleagues in the empirical sciences can do very little to assist us in this regard. Indeed, this is why the necessity of the identity statement "Water $=\mathrm{H}_{2} \mathrm{O}$ ", if the identity is necessary, has to be established by a priori means.

I do not have an a priori proof at hand which would establish the essentiality of composition for chemical substances. I suspect that such a proof is available though and my guess is that it will have something to do with the laws that govern the forming of macrophysical objects. The manner in which subatomic particles form atoms and atoms bind together to form molecules is not arbitrary and it seems plausible to think that, had fundamental forces been arranged otherwise,

16 As opposed to, say, food (cf. Bealer, 1987, p. 296). 
macrophysical objects would not be possible at all. This is because even the slightest change in these forces would cause instability - perhaps the Pauli Exclusion Principle would not hold and electrons would end up in the same quantum state, rendering bonds between atoms impossible. Perhaps the nuclei of atoms would not hold together because the strong force that overpowers the repulsive forces between quarks would not be quite strong enough. Granted, all this is very speculative, but it seems to be the only way to go if we hope to establish the essentiality of composition for chemical substances. It seems to me that the likeliest candidate responsible for the essentiality of composition is the Pauli Exclusion Principle. Although I have no means to prove it here, I think that this principle is a very likely candidate for a metaphysically necessary law of physics.

It should be clear by now what my preferred analysis of modal truths is. I most certainly do not wish to ground modal truths to conceptual truths. As we saw, this is the line that Sidelle, Jackson and Chalmers would perhaps take. I wish to ground modal truths to essential truths, following Kit Fine, i.e., it is the essence of water that makes it necessary that "Water $=\mathrm{H}_{2} \mathrm{O}$ ", if anything. Or, more accurately, it is the essence of chemical substances, if anything, that makes it necessary that "Water $=\mathrm{H}_{2} \mathrm{O}$ ", as the key assumption is that chemical substances have their molecular structures essentially. This line may or may not be different from the Kripkean line; that makes little difference to me. What I wish to emphasise is that the truthmakers of modal claims are essences, not concepts.

Obviously, matters are a lot more complicated than it is often suggested. It is not easy to determine when an identity statement holds by necessity. The origin of these problems seems to be a misconception concerning the "logic of essence", as Lowe puts it (2007, p. 291): in the Kripkean picture essences are apparently derived from semantic intuitions rather than from metaphysical considerations, and thus provide illegitimate grounds for modal truths. The ease with which the purported necessity of certain identity statements has been established is suspicious at the very least, and it appears that one reason for this unwarranted lapse in critical analysis is the ambiguity over the semantic and metaphysical agendas in Kripke's Naming and Necessity. However, it could of course turn out that, despite these shortcomings, most or all of the usual examples of a posteriori necessities are indeed necessary, but perhaps we have been too hasty to conclude that they are also a posteriori. The best course of action to settle the matter is to analyse these cases rigorously in the manner suggested above.

\section{References}

BeAler, G. (1987) “The Philosophical Limits of Scientific Essentialism.” In J. E. Tomberlin (ed.) Philosophical Perspectives 1: Metaphysics, pp. 289-365. Atascadero, CA: Ridgeview. 
Chalmers, D. (1996) The Conscious Mind: In Search of a Fundamental Theory. Oxford: Oxford University Press.

FINE, K. (1994) “Essence and Modality.” In J. E. Tomberlin (ed.) Philosophical Perspectives 8: Logic and Language, pp. 1-16. Atascadero, CA: Ridgeview.

Hughes, C. (2004) Kripke: Names, Necessity, and Identity. Oxford: Oxford University Press.

JaCKson, F. (1998) From Metaphysics to Ethics: A Defence of Conceptual Analysis. Oxford: Oxford University Press.

KRIPKE, S. (1971) "Identity and Necessity." In M. K. Munitz (ed.) Identity and Individuation, pp. 135-164. New York: New York University Press.

KRIPKE, S. (1980) Naming and Necessity. Cambridge, MA: Harvard University Press.

Lowe, E. J. (2007) "A Problem for A Posteriori Essentialism Concerning Natural Kinds." Analysis, 67(4): 286-292.

Salmon, N. U. (2005) Reference and Essence (second edition). New York: Prometheus Books.

Sidelle, A. (2002) “On the Metaphysical Contingency of Laws of Nature.” In T. S. Gendler and J. Hawthorne (eds) Conceivability and Possibility, pp. 309-336. Oxford: Oxford University Press.

Sonmes, S. (2005) Reference and Description: The Case against Two-Dimensionalism. Princeton: Princeton University Press.

SoAmeS, S. (2007) "The Substance and Significance of the Dispute over TwoDimensionalism." Philosophical Books, 48: 34-49.

TAнко, T. E. (2008) "A New Definition of A Priori Knowledge: In Search of a Modal Basis." Metaphysica, 9(2): 57-68. 